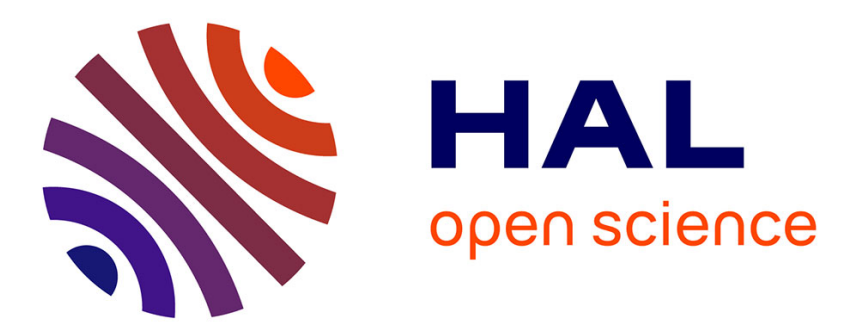

\title{
Graph-Based Motion-Driven Segmentation of the Carotid Atherosclerotique Plaque in 2D Ultrasound Sequences
}

Aimilia Gastounioti, Aristeidis Sotiras, Konstantina S. Nikita, Nikos Paragios

\section{- To cite this version:}

Aimilia Gastounioti, Aristeidis Sotiras, Konstantina S. Nikita, Nikos Paragios. Graph-Based MotionDriven Segmentation of the Carotid Atherosclerotique Plaque in 2D Ultrasound Sequences. Medical Image Computing and Computer-Assisted Intervention - MICCAI 2015, Oct 2015, Munich, Germany. 10.1007/978-3-319-24574-4_66 . hal-01270448

\section{HAL Id: hal-01270448 \\ https://hal.inria.fr/hal-01270448}

Submitted on 9 Feb 2016

HAL is a multi-disciplinary open access archive for the deposit and dissemination of scientific research documents, whether they are published or not. The documents may come from teaching and research institutions in France or abroad, or from public or private research centers.
L'archive ouverte pluridisciplinaire HAL, est destinée au dépôt et à la diffusion de documents scientifiques de niveau recherche, publiés ou non, émanant des établissements d'enseignement et de recherche français ou étrangers, des laboratoires publics ou privés. 


\title{
Graph-based Motion-driven Segmentation of the Carotid Atherosclerotique Plaque in 2D Ultrasound Sequences
}

\author{
Aimilia Gastounioti ${ }^{1,2, *}$, Aristeidis Sotiras ${ }^{2}$, Konstantina S. Nikita $^{1}$, and Nikos \\ Paragios $^{3}$ \\ ${ }^{1}$ Biomedical Simulations \& Imaging Laboratory, School of Electrical and Computer \\ Engineering, National Technical University of Athens, Athens, Greece \\ gaimilia@biosim.ntua.gr, knikita@ece.ntua.gr \\ ${ }^{2}$ Center for Biomedical Image Computing and Analytics, Department of Radiology, \\ University of Pennsylvania, Philadelphia, USA \\ sotar22@gmail.com \\ ${ }^{3}$ Center for Visual Computing, Department of Applied Mathematics, Ecole Centrale \\ de Paris, Paris, France \\ nikos.paragios@ecp.fr
}

\begin{abstract}
Carotid plaque segmentation in ultrasound images is a crucial step for carotid atherosclerosis. However, image quality, important deformations and lack of texture are prohibiting factors towards manual or accurate carotid segmentation. We propose a novel fully automated methodology to identify the plaque region by exploiting kinematic dependencies between the atherosclerotic and the normal arterial wall. The proposed methodology exploits group-wise image registration towards recovering the deformation field, on which information theory criteria are used to determine dominant motion classes and a map reflecting kinematic dependencies, which is then segmented using Markov random fields. The algorithm was evaluated on 120 cases, for which manually-traced plaque contours by an experienced physician were available. Promising evaluation results showed the enhanced performance of the algorithm in automatically segmenting the plaque region, while future experiments on additional datasets are expected to further elucidate its potential.
\end{abstract}

Keywords: segmentation; carotid plaque; image registration; ultrasound

\section{Introduction}

Carotid plaques are buildups of fatty substances, scar tissue and cholesterol deposits in the inner lining of the carotid artery and they are caused by a chronic degenerative arterial disease called atherosclerosis. The direct association of these arterial lesions with stroke events and the limitations of the current risk-stratification practice both underline the need for the development of computer-aided-diagnosis (CAD) systems for carotid plaques [1]. In particular, the development of effective CAD schemes, which are based on B-mode ultrasound (US) image analysis, is considered a grand challenge by the scientific 
community, due to the fact that this modality holds a prominent position in screening and follow-up exams of patients with carotid atherosclerosis. In this line of work, and given that manual tracing of regions of interest is always a timeconsuming and strenuous task, automated plaque segmentation in B-mode US is a critical step towards user-independent measurements of novel biomarkers: (a) total plaque area [2], (b) plaque surface irregularities [3], (c) plaque deformations during the cardiac cycle [4], and (d) the underlying material composition of the plaque (texture descriptors) [1].

An attempt to provide a short overview of related studies [5]-[10] is presented in Table 1. It should be noted that there is quite limited work on the segmentation of the carotid plaque in 2D B-mode US of longitudinal sections of the carotid artery. Recent work explore either well known and well studied active-contours on the image features domain [5] or a conventional Bayesian formulation [7]. Full automatization is achieved only in [5]. It is also important to note that both approaches are applied separately to each frame of the sequence and they are based on local intensity values to segment the carotid plaque; the deformation field is used either to initialize the segmentation process in subsequent frames [5] or to ensure cohesion between segmentation results in different time points [7]. However, US is characterized by low image quality and texture features may vary significantly over different devices, image settings, and operators. Moreover, a frame-wise concept of segmenting the carotid plaque may limit the overall performance of the algorithm due to image artifacts in isolated frames, the view point of the sensor, the important displacements, and the variations of image intensities; it can also be more time-consuming than simultaneously using all frames (one-shot approach).

Table 1: Previous work on developing computerized methodologies for segmenting the carotid plaque in B-mode ultrasound.

\begin{tabular}{||cccc||}
\hline \hline Study & Sample & Imaging modality & Fully Automatic \\
\hline \hline$[5]$ & 43 & 2D B-mode video - Longitudinal view & $\sqrt{ }$ \\
{$[6]$} & 7 & 2D B-mode image - Transverse view & $\times$ \\
{$[7]$} & 33 & 2D B-mode video - Longitudinal view & $\times$ \\
{$[8]$} & 1 & 3D B-mode image - Transverse view & $\sqrt{ }$ \\
{$[9]$} & 21 & 3D B-mode video - Transverse view & $\times$ \\
{$[10]$} & 22 & 3D B-mode image - Longitudinal \& Transverse view & $\times$ \\
\hline
\end{tabular}

In this study, we aim to tackle aforementioned limitations and, motivated by recent findings suggesting that the kinematic activity of the carotid plaque differs from that of non-atherosclerotic arterial-wall segments [4], we introduce a novel fully automated one-shot segmentation approach, the core of which is the exploitation of motion as segmentation feature. The algorithm relies on groupwise image registration towards recovering the deformation field, on which in- 
formation theory criteria are used to determine dominant motion classes and a map reflecting kinematic dependencies, which is then segmented using Markov random fields (MRFs). The designed algorithm is evaluated on $120 \mathrm{~B}$-mode US video recordings of patients with carotid atherosclerosis.

\section{The Proposed Methodology}

The use of motion as segmentation feature involves three challenges; (i) the estimation of the deformation vectors, (ii) the identification of dominant kinematic hypotheses, and the (iii) segmentation of the image domain according to these hypotheses.

The proposed approach aims to explore motion differences as a feature vector towards carotid segmentation. Such an approach can either be addressed through a sequential approach, where either optical flow is estimated sequentially or all frames are mapped to a reference frame. The first approach is proned to accumulation of errors, which can be critical if motion vectors are used as segmentation features, while the latter can be quite sensitive in the presence of important displacements throughout the sequence while it introduces also a significant bias depending on the choice of the reference frame. Recent studies have shown that population (group-wise) registration methods perform in general more reliably compared to the ones exploiting the pair-wise assumption.

\subsection{Group-wise Image Registration}

In this step, the deformation field is estimated by registering the entire image sequence through an one-shot optimization approach. Let us now consider without loss of generality a sequence of $N$ frames where each frame is deformed according to a deformation model $T_{i}$. Inspired from the ideas presented in [11], we adopt a discrete optimization framework which aims to estimate all deformations at a single step. The central idea is to deform the grids simultaneously, such that meaningful correspondences between the $N$ images are obtained and their mapping to the geometry (reference pose, $R$ ) creates a statistically compact variable. The described graphical model is expressed in the form of a MRF and the objective function in an abstract level can be expressed as follows:

$$
\begin{aligned}
& E\left(T_{i}, R\right)=\alpha \sum_{i=1}^{N} \sum_{j=i+1}^{N} \iint_{\Omega} \rho\left(I_{i}\left(T_{i}\right), I_{j}\left(T_{j}\right)\right) d \omega+ \\
& \beta \sum_{i=1}^{N} \iint_{\Omega} \psi\left(T_{i}\right) d \omega+\gamma \iint_{\Omega} \eta\left(R, I_{1}\left(T_{1}\right), \cdots, I_{N}\left(T_{N}\right)\right)
\end{aligned}
$$

seeking to optimize an objective function which consists of three terms weighted by factors $\alpha, \beta$, and $\gamma$; one that measures the similarity $(\rho)$ between two images, a second term which incorporates a convex function, $\psi$, imposing smoothness at each deformation field and a third term which measures the similarity, $\eta$, 
of the images on the intersection of the deformed images to enforce pair-wise correspondences.

This is a coupled optimization problem that is discretized towards reducing complexity. Deformations are expressed through reduced complexity grids endowed with an interpolation strategy (free-form deformations), while reference image intensities are discretized as well. The optimization is, then, performed with the efficient linear programming method which is described in detail in [12]. The outcome of this inference process consists of $N$-deformation models mapped all to a common pose and reference frame where each pixel is associated with an $N \times 2$-dimensional feature vector (vertical and horizontal displacements towards the estimated reference space). Reasoning on this space requires estimation of the dominant hypotheses.

\subsection{A Mutual-Information Map Reflecting Kinematic Dependencies}

Let us denote the resultant of an $N \times 2$-dimensional feature vector as MTD (magnitude of total displacement). In this step, each pixel is associated with an MTD vector and the goal is to identify dominant motion classes and elucidate kinematic dependencies among pixels. We empirically found that the application of independent component analysis (ICA) on the MTD vector set mostly captured the motion of the plaque, with a number of 50 independent components sufficiently explaining variance in kinematic activity. Therefore, a subset of $K=50$ independent MTD vectors (IVs) are first isolated using ICA.

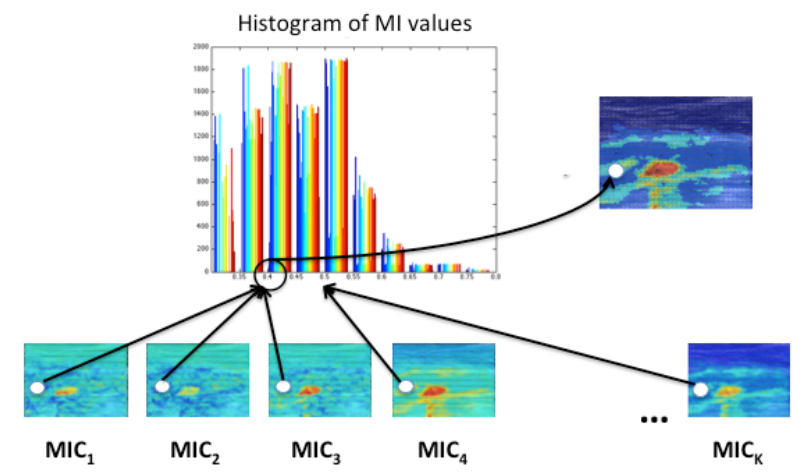

Fig. 1: Combining $K$ mutual information color maps (MIC), each representing the mutual information (in [0: blue - 1: red]) between an independent vector and MTD vectors, to estimate a final mutual information value for each pixel.

Having isolated motion vectors that mainly describe plaque motion, the next step is to discriminate regions where the kinematic activity mostly depends on those vectors. To this end, each MTD vector is mapped to the IVs (ICA base) using the mutual information (MI), which was empirically found to perform 
better than simple projection to the ICA base, as follows. $K$ colormaps, each representing the MI values of every MTD vector with the $k$ th $\operatorname{IV}(k \in[1, K])$, are produced and a final MI value is attributed to each pixel through majority voting. Specifically, based on the histogram of all $K$ color maps, each pixel is associated with the center of the bin where the majority of the corresponding MI values belong (Fig. 1).

\subsection{Graph-Based Segmentation of the Mutual-Information Map}

At this stage, the final MI map is segmented by following again the concepts of graphical models and MRFs. A grid is superimposed onto the map, with each pixel corresponding to a node and the edge system is constructed by assuming an 8-neighbor connectivity for smoother segmentation results (Fig. 2), giving rise to a binary labeling problem (label 1: "plaque" and label 2: "non plaque").

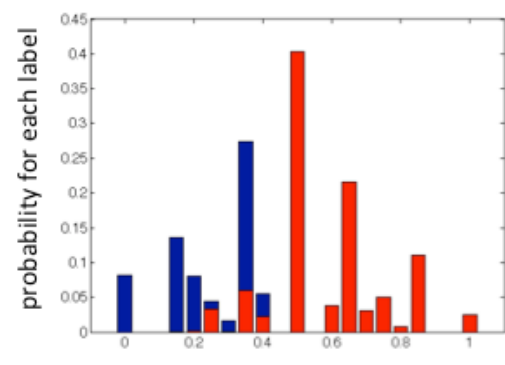

MI map value

label 1: plaque label 2: not plaque

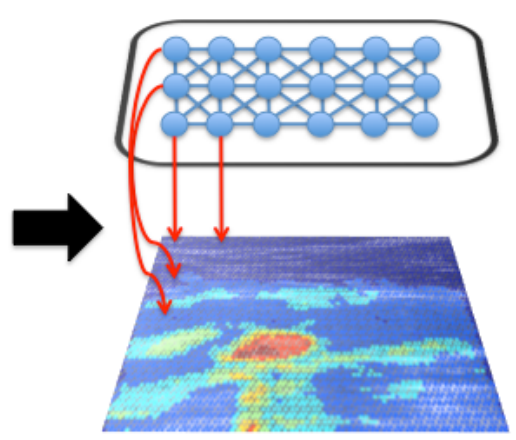

Fig. 2: (Right) Rough presentation of the underlying graph which is used in graph-based segmentation of the final color map; for simplicity reasons, only a subset of nodes and edges is displayed. The red edges represent the relationship between the grid nodes and the pixels of the color map, while the blue ones correspond to the pairwise potentials. The singleton potentials are given by the likelihood of a label given the MI map value (bar chart on the left).

The objective function to be optimized consists of two terms, one singleton representing the node-labeling costs $(\overline{\mathbf{g}})$ and one pair-wise standing for the costs to split neighboring nodes $(\overline{\mathbf{f}})$ :

$$
\varepsilon(\overline{\mathbf{g}}, \overline{\mathbf{f}})=\min _{u} \sum_{p \in V} \bar{g}_{p}\left(u_{p}\right)+\lambda \sum_{(p, q) \in E} \bar{f}_{p q}\left(u_{p}, u_{q}\right)
$$

where $V$ and $E$ denote, respectively, the vertices and edges of the graph. The labeling costs for each node are considered to be proportional to the likelihood of a label given the final MI map value for the corresponding pixel. These likelihood values are already learned in a training step (see also section 3). The 
pair-wise potentials are considered to be equal to 1 and 0 for neighboring pixels with different or the same labels, respectively, assuming equal weights for all connections. The optimization problem is solved again with a previously presented linear programming method [12].

\section{Evaluation Process and Results}

The study dataset consisted of 120 patients with established carotid atherosclerosis; the atherosclerotic lesions were mainly soft plaques causing mild or moderate lumen stenosis, which are more often clinically encountered and prone-torupture. For each subject, the carotid artery was scanned in the longitudinal direction and a B-mode US image sequence was recorded at a rate higher than 25 frames/s for at least one cardiac cycle. The algorithm was empirically parameterized with $32 \times 32$ grids and $\alpha, \beta$ and $\gamma$ equal to 2, 1 and 18, respectively, for the group-wise image registration. Optimal graph-based segmentation of the final MI maps was achieved with weights equal to 1 for all connections.

The algorithm was evaluated on the dataset following leave-one-out cross validation, by repeating the following phases for each patient $m$ (with $m \in$ $[1,120])$ considering that the $m^{t h}$ patient was the testing case and the other patients formed the training set. In the training phase, the final MI maps for the training cases, together with the ground-truth plaque regions (as manually traced by an experienced vascular physician), were fed to Adaboost in order to learn the likelihood distributions of each label over different MI values. Then, the algorithm was applied to the testing case and the agreement between the segmentation result and the corresponding ground-truth plaques was measured in terms of the true negative (TNF) and the true positive (TPF) fractions, the Dice coefficient, the overlap index, and the mean contour distance (MCD). In all repetitions, the "plaque" label was associated with statistically higher MI values than the "non plaque" label (Wilcoxon rank-sum test, $p<0.05$ ).
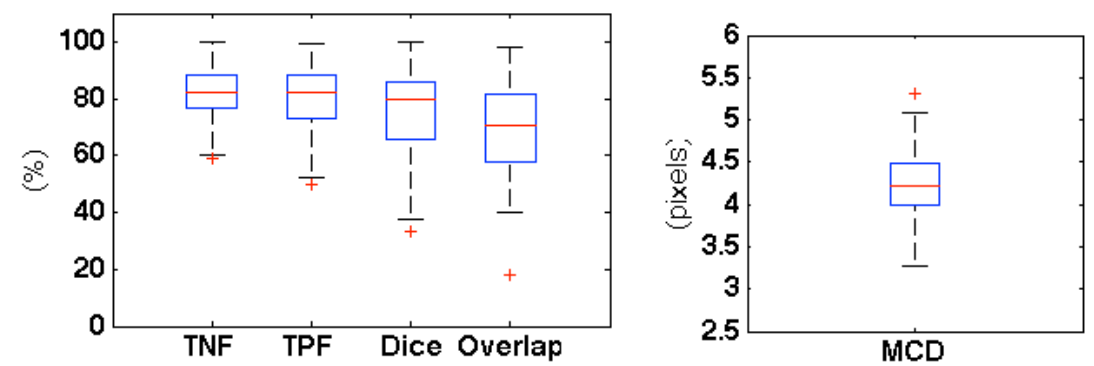

Fig. 3: Boxplots of the evaluation results for all patients.

The evaluation results for all patients are summarized in the boxplots of fig. 3. Fig. 4 illustrates examples of the segmentation results and the corresponding 
ground-truth boundaries. The evaluation results are in line with those presented in the limited number of related studies which are available in the literature $[5,7]$. Although straightforward comparisons are not possible due to different datasets, the promising performance of the proposed algorithm is further enhanced considering that (a) the algorithm achieved similar segmentation accuracy on a 3-times larger database (Table 1), (b) it uses motion, which is more reliable and more robust over different US image recordings than texture, as segmentation feature, and (c) it is an one-shot approach which, as explained in section 1, limits many drawbacks of US imaging. Moreover, it is important to note that plaque segmentation in US is a very challenging task, with low agreement even in manual delineation by expert operators [13].
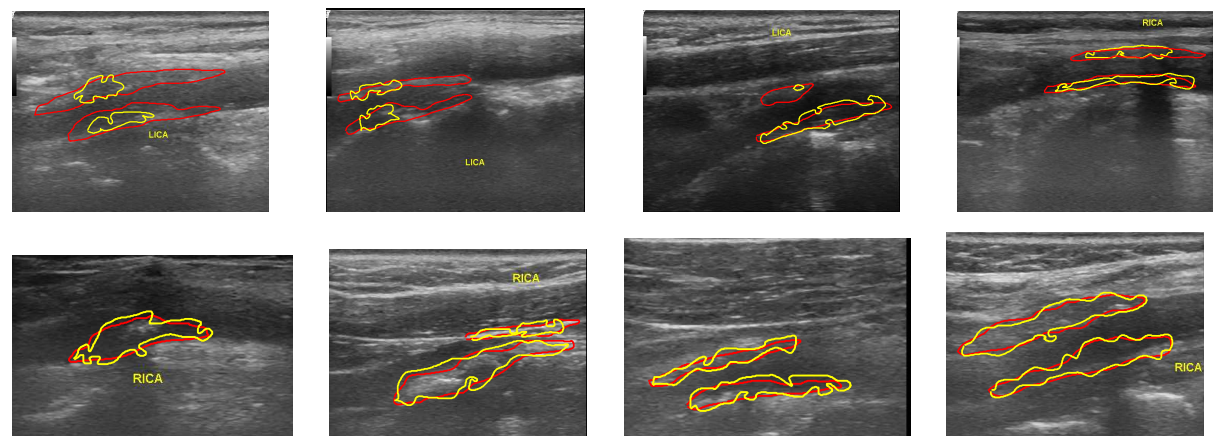

Fig. 4: Outlines of plaque regions as estimated by the proposed algorithm (yellow) and as manually traced by experienced vascular physicians (red) for cases of minimum (1st row, $\mathrm{TNF}=60-65 \%, \mathrm{TPF}=61-64 \%$, Dice $=45-52 \%$, Overlap $=40$ $50 \%, \mathrm{MCD}=4.5-5.0$ pixels) and maximum performance (2nd row, $\mathrm{TNF}=82-87 \%$, $\mathrm{TPF}=80-85 \%$, Dice $=79-81 \%$, Overlap $=75-80 \%, \mathrm{MCD}=3.5-4.0$ pixels $)$ of the al gorithm.

\section{Discussion}

This study introduced a fully automated algorithm for graph-based motiondriven segmentation of the carotid plaque in 2D ultrasound image sequences. Future experiments, including datasets of different US scanners and scanner settings, as well as ground-truth plaque outlines by multiple experienced physicians, are expected to further elucidate the potential of the algorithm. In a future perspective, integration of visual and motion features within the segmentation framework is a natural extension of the proposed framework. In terms of methodological view point, integration of motion hypotheses generation along with segmentation can be done jointly if the task is considered as an unsupervised clustering problem where both assignments, number of clusters and cluster 
centers are unknown. Furthermore, the entire chain can be considered as a single optimization problem if the "segmentation" labels are integrated to the population registration framework a "singleton" likelihoods acting on the reconstructed reference image in the form of a higher order potential. This will allow - given a predefined set of motion hypotheses - to jointly register and segment all images at once and eventually will improve both segmentation and registration results since their solutions will mutually benefit from their association.

\section{References}

1. Golemati, S., Gastounioti, A., Nikita, K.S.: Toward novel noninvasive and low-cost markers for predicting strokes in asymptomatic carotid atherosclerosis: the role of ultrasound image analysis. IEEE Trans Biomed Eng (Special issue on grand challenges in engineering life sciences and medicine). 60(3): 652-658 (2013)

2. Destrempes, F., Meunier, J., Giroux, M.F., Soulez, G., Cloutier, G.: Segmentation of plaques in sequences of ultrasonic b-mode images of carotid arteries based on motion estimation and a Bayesian model. IEEE Trans Biomed Eng. 58(8): 2202-2211 (2011)

3. Prabhakaran, S., Rundek, T., Ramas, R., Elkind, M.S., Paik, M.C., Boden-Albala, B., Sacco, R.L.: Carotid plaque surface irregularity predicts ischemic stroke: the northern Manhattan study. Stroke. 37(11): 2696-2701 (2006)

4. Gastounioti, A., Golemati, S., Stoitsis, J.S., Nikita, K.S.: Adaptive block matching methods for carotid artery wall motion estimation from B-mode ultrasound: in silico evaluation \& in vivo application. Phys Med Biol. 58: 8647-8661 (2013)

5. Loizou, C., Petroudi, S., Pantziaris, M., Nicolaides, A., Pattichis, C.: An integrated system for the segmentation of atherosclerotic carotid plaque ultrasound video. IEEE Trans Ultrason Ferroelectr Freq Control. 61(1): 86-101 (2014)

6. Mao, F., Gill, J., Downey, D., Fenster, A.: Segmentation of carotid artery in ultrasound images: Method development and evaluation technique. Med Phys. 27(8): 1961-1970 (2000)

7. Destrempes, F., Meunier, J., Giroux, M.F., Soulez, G., Cloutier, G.: Segmentation of plaques in sequences of ultrasonic b-mode images of carotid arteries based on motion estimation and a Bayesian model. IEEE Trans Biomed Eng. 58(8): 2202-2211 (2011)

8. Zahalka, A., Fenster, A.: An automated segmentation method for three-dimensional carotid ultrasound images. Phys Med Biol. 46(4): 1321-1342 (2001)

9. Ukwatta, E., Awad, J., Ward, A.D., Buchanan, D., Samarabandu, J., Parraga, G., Fenster, A.: Three-dimensional ultrasound of carotid atherosclerosis: semiautomated segmentation using a level set-based method. Med Phys. 38(5): 2479-2493 (2011)

10. Buchanan, D., Gyacskov, I., Ukwatta, E., Lindenmaier, T., Fenster, A., Parraga: Semi-automated segmentation of carotid artery total plaque volume from three dimensional ultrasound carotid imaging. Proc. SPIE. 8317: 83170I-83170I-7 (2012)

11. Sotiras, A., Komodakis, N., Glocker, B., Deux, J.-F., Paragios, N.: Graphical models and deformable diffeomorphic population registration using global and local metrics. In: MICCAI (2009)

12. Komodakis, N., Tziritas, G., Paragios, N.: Performance vs computational efficiency for optimizing single and dynamic mrfs: Setting the state of the art with primal-dual strategies. Comput Vis Imag Und 112(1): 14-29 (2008)

13. Polak, J.F., Pencina, M.J., OLeary, D.H., DAgostino, R.B.: Common-carotid artery intima-media thickness progression as a predictor of stroke in multi-ethnic study of atherosclerosis. Stroke 42(11): 3017-3021 (2011) 